\title{
Algebraic-Phase Scrambling Sequences for Code-Spread Code-Division Multiple-Access
}

\author{
Yi Hong \\ Institute of Advanced Telecom. \\ University of Wales, Swansea, UK \\ y.hongeswansea.ac.uk
}

\author{
Shlomo Shamai (Shitz) \\ Technion-Israel Institute of Technology \\ Technion City, Haifa 32000, Israel \\ sshlomodee.technion.ac.il
}

\author{
Emanuele Viterbo \\ DEIS-Università della Calabria \\ Rende CS 89036, Italy \\ viterbo@deis.unical.it
}

\begin{abstract}
In this paper, we first present a modified codespread code-division multiple-access (CS-CDMA) scheme, named phase-scrambling CDMA (PS-CDMA), for a Gaussian multiple access channel (MAC). In PS-CDMA, we realize the codespreading using a low-rate serially concatenated code consisting of a convolutional code and a repetition code. Then, we use complex user-specific algebraic-phase scrambling sequences to distinguish users. The receiver is based on the iterative multiuser decoding suggested in $[5,8]$. Next, we design complex algebraicphase scrambling sequences to mitigate multiple access interferences for overloaded PS-CDMA, i.e., the number of users is greater than the spreading factor. By analyzing extrinsic information transfer (EXIT) curves and the trajectories, we demonstrate that PS-CDMA using the proposed scrambling sequences has faster iterative decoding convergency and better system performance, when compared to the PS-CDMA using random-phase scrambling sequences and some previously known multiple access schemes.
\end{abstract}

\section{INTRODUCTION}

In code-division multiple-access (CDMA), Viterbi and Hui $[1,2]$ independently proposed the idea of using low-rate codes alone for bandwidth expansion in order to achieve the maximum theoretical performance of the multiple access system.

Motivated by the above, authors of [3] proposed codespread CDMA (CS-CDMA) by making use of a class of lowrate convolutional codes [4], followed by ideal interleavers, user-specific pseudorandom scrambling sequences and harddecision decoding combined with interference cancellation. Recently, a chip-interleaved CDMA was proposed in [7], which admits a transmitter structure similar to that of CSCDMA, but with user-specific interleavers to distinguish users. At the receiver, a joint soft-input soft-output (SISO) iterative multiuser decoding was adopted. This multiple access scheme was also proposed in [8] under the name of interleave-division multiple-access (IDMA).

In this paper, we present a modified CS-CDMA, named phase-scrambling CDMA (PS-CDMA), over a Gaussian multiple access channel (MAC). At the transmitter of each user, we use a low-rate serially concatenated code consisting of a convolutional code and a repetition code for code-spreading. Then, we use complex user-specific algebraic-phase scrambling sequences to distinguish users from each other. At the receiver, we employ a joint iterative soft-input soft-output (SISO) MMSE-filtered parallel interference cancellation (PIC) multiuser detection (MUD) [5,8], phase-remover, and channel decoders.

Conventional complex scrambling sequences were mainly designed for asynchronous CDMA with the aim of low autocorrelation sidelobes and low crosscorrelation [9-11]. Unlike these sequences in $[9,11]$, in our paper, we aim at designing complex scrambling sequences for a synchronous overloaded PS-CDMA, i.e., the number of users is greater than the spreading-sequence length. In particular, we construct such sequences to mitigate multiple access interferences (MAIs) for overloaded PS-CDMA. By analyzing extrinsic information transfer (EXIT) charts and trajectories, we show that PSCDMA using the proposed scrambling sequences has faster iterative decoding convergence and better bit error rate (BER) performance, when compare to that using random-phase scrambling sequences and IDMA [7,8]. Finally, we remark that our scrambling sequence design can be also easily adapted to any multiple access system with different number of users and various spreading factors.

The outline of this paper is organized as follows. Section II introduces the proposed PS-CDMA. In Section III we present the design of algebraic-phase scrambling sequences for PSCDMA. In Section, we compare EXIT charts and trajectories, as well as the BER performance of PS-CDMA using different scrambling sequences and IDMA systems. Finally, conclusions are drawn in Section V.

Notations: Let $\mathbf{E}[\cdot]$ and $\operatorname{Var}[\cdot]$ denote mean and variance of a random variable. Let $\Re(\cdot)$ be the real part of a complex number.

\section{PS-CDMA}

We consider a synchronous CS-CDMA system with $K$ users transmitting with equal power over a MAC using BPSK modulation. Fig. 1(a) shows the transmitter structure for user $k$, where $k=1, \ldots, K$. The input data sequence $\mathbf{d}_{k}=$ $\left[d_{k}(1), \ldots, d_{k}(i), \ldots, d_{k}\left(N_{b}\right)\right]$, is encoded by a convolutional code with rate $R_{1}$, producing $\mathbf{b}_{k}=\left[b_{k}(1), \ldots, b_{k}(l), \ldots\right.$, $\left.b_{k}(L)\right]$. We note that $N_{b}=R_{1} L$. The convolutionally encoded sequence $\mathbf{b}_{k}$ is further encoded (spreaded) by a low-rate repetition code with rate $R_{2}=1 / S$, generating $\mathbf{c}_{k}=\left[c_{k}(1)\right.$, $\left.\ldots, c_{k}(j), \ldots, c_{k}(J)\right]$, where $J=S L$. The coded sequence $\mathbf{c}_{k}$ is multiplied by an algebraic-phase scrambling sequence, i.e., user- and chip-specific phase rotation $\left\{e^{j \theta_{i}^{(k)}}\right\}, k=1, \ldots, K$, 


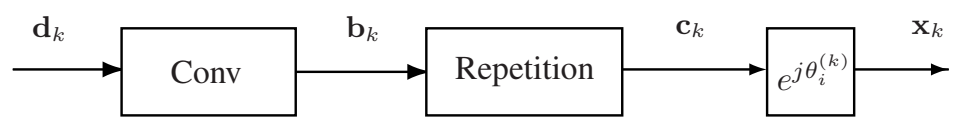

(a)

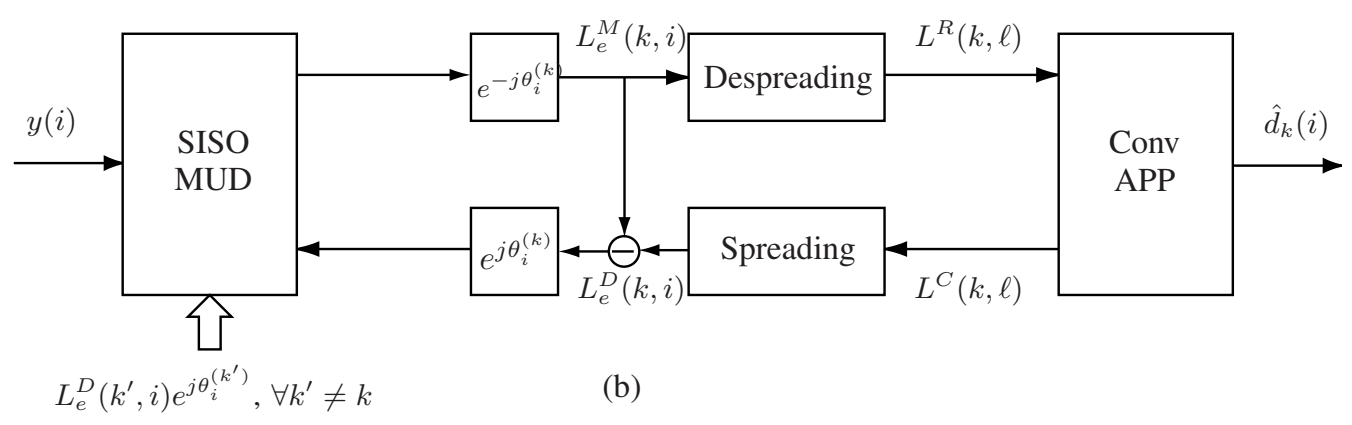

Fig. 1. The PS-CDMA transmitter (a) and receiver (b) structures for user $k$.

$i=1, \ldots, J$, producing $\mathbf{x}_{k}=\left[x_{k}(1), \ldots, x_{k}(J)\right]$, where we denote the components of $\mathbf{x}_{k}$ as chips and the condition $J>K$ is assumed in the paper. We call such a multiple access scheme phase-scrambling CDMA (PS-CDMA). The system load of PS-CDMA is defined as $\alpha=\frac{K}{S}$ [6].

At the receiver chip-matched filtering is performed, providing a sequence of chip-level observables,

$$
y(i)=\sum_{k=1}^{K} h_{k}(i) x_{k}(i)+n(i), \quad i=1, \ldots, J,
$$

where $h_{k}(i)$ is the channel coefficient for user $k$ at chip $i$ and $n(i)$ denotes the sample of an additive white Gaussian noise (AWGN) process with variance $\sigma^{2}=N_{0} / 2$. A Gaussian MAC is assumed in the paper, i.e., $h_{k}(i)=1, \forall k$ and $\forall i$.

In PS-CDMA systems, we employ user-specific and chipspecific phase rotations, i.e., $e^{j \theta_{i}^{(k)}} \neq e^{j \theta_{i^{\prime}}^{\left(k^{\prime}\right)}}$ for $k \neq k^{\prime}$ and $i \neq i^{\prime}$. Assuming the phase-rotations are generated independently for all users, the adjacent chips in the sequence $\mathbf{x}^{(k)}$ are approximately uncorrelated. Therefore a simplified version of the SISO MMSE-filtered PIC MUD in [5] can be adopted at the receiver.

The receiver structure for user $k$ is shown in Fig. 1(b), where the MUD is concatenated with one phase-remover and two $a$ posteriori probability (APP) channel decoders for repetition code (despreading) and convolutional code, respectively. The multiple-access constraints and code constraints are considered separately by the MUD, the phase-remover, and the channel decoders.

Following the derivations in $[7,8]$, the received signal in (1) can be rewritten as

$$
\begin{aligned}
y(i) & =h_{k}(i) x_{k}(i)+\sum_{k^{\prime} \neq k} h_{k^{\prime}}(i) x_{k^{\prime}}(i)+n(i) \\
& =h_{k}(i) x_{k}(i)+V_{k}(i),
\end{aligned}
$$

where

$$
V_{k}(i) \triangleq \sum_{k^{\prime} \neq k} h_{k^{\prime}}(i) x_{k^{\prime}}(i)+n(i)
$$

is the sum of the multiple-access interference and noise terms of the received sample $y(i)$, when detecting user $k$. For a large number of users, it follows from the central limit theorem that $V_{k}(i)$ is approximately Gaussian distributed. Let us define

$$
\mathbf{E}[y(i)] \triangleq \sum_{k=1}^{K} h_{k}(i) \mathbf{E}\left[x_{k}(i)\right]
$$

and

$$
\operatorname{Var}[y(i)] \triangleq \sum_{k=1}^{K}\left|h_{k}(i)\right|^{2} \operatorname{Var}\left[x_{k}(i)\right]+\sigma^{2}
$$

It follows that $V_{k}(i)$ has mean

$$
\mu_{k}(i) \triangleq \mathbf{E}\left[V_{k}(i)\right]=\mathbf{E}[y(i)]-h_{k}(i) \mathbf{E}\left[x_{k}(i)\right]
$$

and variance

$$
\nu_{k}(i) \triangleq \operatorname{Var}\left[V_{k}(i)\right]=\operatorname{Var}[y(i)]-\left|h_{k}(i)\right|^{2} \operatorname{Var}\left[x_{k}(i)\right]
$$

The joint SISO MUD and phase-remover are employed to calculate the extrinsic log-likelihood ratio (LLR) of the coded bit $c_{k}(i)$ using [5]

$$
\begin{aligned}
L_{e}^{M}(k, i) & =\log \frac{p\left(y(i) \mid c_{k}(i)=+1\right)}{p\left(y(i) \mid c_{k}(i)=-1\right)} \\
& =2 h_{k}(i) \Re\left(\frac{y(i)-\mu_{k}(i)}{\nu_{k}(i)} e^{-j \theta_{i}^{(k)}}\right) \\
& =2 h_{k}(i) \Re\left(\frac{z_{k}(i)}{\nu_{k}(i)} e^{-j \theta_{i}^{(k)}}\right)
\end{aligned}
$$

and $z_{k}(i)=y(i)-\mu_{k}(i), i=1, \ldots, J$. The extrinsic LLRs $L_{e}^{M}(k, i), \forall i$ are further forwarded to the SISO channel decoder. 
At the receiver for user $k$, we employ the two APP decoders mentioned above. Considering decoding of the repetition code, recall that for the convolutionally encoded bit $b_{k}(1)$, we have $c_{k}(j)=b_{k}(1)$ for $j=1, \ldots, S$. We can then compute the $a$ posteriori LLR of $d_{k}(1)$ as

$$
L^{R}(k, 1)=\sum_{j=1}^{S} L_{e}^{M}(k, j)
$$

The APP decoder of the repetition code can be seen as a despreading operation, and in the same manner, we compute $L^{R}(k, \ell), \ell=1, \ldots, L$. Then the convolutional SISO decoder computers the LLRs $L^{C}(k, \ell)$ of the information bits $d_{k}(\ell)$.

Given the first bit $d_{k}(1)$ of user $k$, the a posteriori LLR $L^{C}(k, 1)$ is repeated $S$ times to perform the spreading operation. We then compute the extrinsic LLRs of the corresponding coded bits $c_{k}(j), j=1, \ldots, S$, using

$$
L_{e}^{D}(k, j)=L^{C}(k, 1)-L_{e}^{M}(k, j) j=1, \ldots, S .
$$

In the same manner, we compute all the extrinsic LLRs $L_{e}^{D}(k, i), i=1, \ldots, S L$. The above extrinsic LLRs $L_{e}^{D}(k, i), \forall i$ are further multiplied by phase-rotations, updating the a priori LLRs in the SISO MUD block using

$$
\mathbf{E}\left[x_{k}(i)\right]=h_{k}(i) \cdot \tanh \left(L_{e}^{D}(k, i) / 2\right) \cdot e^{j \theta_{i}^{(k)}}
$$

and

$$
\operatorname{Var}\left[x_{k}(i)\right]=\left|h_{k}(i)\right|^{2}-\left|\mathbf{E}\left[x_{k}(i)\right]\right|^{2}
$$

Note that at the first iteration, assuming equally likely chips, we have $\mathbf{E}\left[x_{k}(i)\right]=0$ and $\operatorname{Var}\left[x_{k}(i)\right]=1, k=1, \ldots, K$, $i=1, \ldots, J$. This completes one iteration of the joint SISO detection and decoding process.

\section{Phase-Rotation ScRAmbling Sequences Design}

Let us consider a $K \times J$ phase-scrambling sequence matrix

$$
\boldsymbol{\Phi} \triangleq\left(\begin{array}{ccc}
e^{j \theta_{1}^{(1)}} & \cdots & e^{j \theta_{J}^{(1)}} \\
\vdots & & \vdots \\
e^{j \theta_{1}^{(k)}} & \cdots & e^{j \theta_{J}^{(k)}} \\
\vdots & & \vdots \\
e^{j \theta_{1}^{(K)}} & \cdots & e^{j \theta_{J}^{(K)}}
\end{array}\right)
$$

We consider two cases:

1) Random-phases: the phases $\theta_{i}^{(k)}$ are selected randomly in the interval $[0,2 \pi]$.

2) Algebraic-phases: the phases are selected as $\theta_{i}^{(k)}=$ $2 \pi s(k-1)(i-1) / n, i=1, \ldots, J$, for some integers $s$ and $n$.

In the latter case, we choose the integers $s, n$ according to the following Lemma.

Lemma 1: Assuming a frame length $J \gg n$ and $s$ coprime to $n$, if $n$ divides $J$ then the matrix $\boldsymbol{\Phi}$ is unitary (i.e., the userspecific phase scrambling codes are orthogonal); if $n$ does not divide $J$ then the user-specific phase scrambling codes are approximately orthogonal.
Proof. Consider the complex scalar product of two rows, $k$ and $k^{\prime}$, of $\boldsymbol{\Phi}$ :

$\gamma=\sum_{i=0}^{J-1} e^{j 2 \pi s(k-1) i / n} e^{-j 2 \pi s\left(k^{\prime}-1\right) i / n}=\frac{e^{j 2 \pi s\left(k-k^{\prime}\right) i J / n}-1}{e^{j 2 \pi s\left(k-k^{\prime}\right) i / n}-1}$

Since $s$ is coprime to $n$, when $J / n$ is an integer we have

$$
\gamma= \begin{cases}0 & k \neq k^{\prime} \\ J & k=k^{\prime}\end{cases}
$$

If $J=q n+r$ for some integers $q$ and $r$, then

$$
\gamma= \begin{cases}\frac{e^{j 2 \pi s\left(k-k^{\prime}\right) i r / n}-1}{e^{j 2 \pi s\left(k-k^{\prime}\right) i / n}-1} & k \neq k^{\prime} \\ q n & k=k^{\prime}\end{cases}
$$

When $J \gg n$ for $k \neq k^{\prime}$ we have $|\gamma|<r \ll q n$, which results in a practically orthogonal matrix $\boldsymbol{\Phi}$ for sufficiently large $J$.

We now analyze the relation between the spreading factor $S$ and the choice on the parameter $n$. In particular, we distinguish two cases: (i) $K<S$ and (ii) $K>S$ (overloaded system). For $K<S$ it is usual to take orthogonal signature waveforms at the bit level. This is achieved by letting $n=S$ which guarantees orthogonality within each coded bit interval of all the users. In the overloaded case $(K>S)$ bit level orthogonality among all signature waveforms cannot be guaranteed, but we observed how the choice of letting $n=a S$ for some integer $a$ is beneficial as illustrated by the following

Lemma 2: Let $n=a S$ for some integer $a$ and consider the matrix $\boldsymbol{\Phi}^{\prime}$ consisting of the first $S$ columns of $\boldsymbol{\Phi}$ in (5) then the $K$ row vectors of $\boldsymbol{\Phi}^{\prime}$ satisfy the following property:

- the row vectors in the set $\mathcal{K}_{a, b}$ with index $1 \leq a \ell+b \leq K$ are mutually orthogonal for a fixed integer $1 \leq b<a$ and $\ell=0,1, \ldots$

- the vectors in different sets $\mathcal{K}_{a, b}$ and $\mathcal{K}_{a, b^{\prime}}$ are not orthogonal.

Proof. Consider the complex scalar product of two rows of $\boldsymbol{\Phi}^{\prime}$ with indexes $k=a \ell+b$ and $k^{\prime}=a \ell^{\prime}+b$ with $\ell \neq \ell^{\prime}$ :

$$
\sum_{i=0}^{n / a-1} e^{j 2 \pi s\left(k-k^{\prime}\right) i / n}=\frac{e^{j 2 \pi s\left(\ell-\ell^{\prime}\right) i}-1}{e^{j 2 \pi s\left(\ell-\ell^{\prime}\right) i a / n}-1}=0
$$

Consider the complex scalar product of two rows of $\boldsymbol{\Phi}^{\prime}$ with indexes $k=a \ell+b$ and $k^{\prime}=a \ell^{\prime}+b^{\prime}$ with $b \neq b^{\prime}$ and any $\ell, \ell^{\prime}$ :

$$
\sum_{i=0}^{n / a-1} e^{j 2 \pi s\left(k-k^{\prime}\right) i / n}=\frac{e^{j 2 \pi s\left(b-b^{\prime}\right) i / a}-1}{e^{j 2 \pi s\left(k-k^{\prime}\right) i / n}-1} \neq 0
$$

In particular, for $a=2$ we find the best performance, since, in the case of $K>S$, the iterative detection of one user will suffer at most from the interference of $K / 2$ other users. In order to illustrate the above design, we present an example as follows.

Example: Under Gaussian MACs, we consider an overloaded PS-CDMA system with $K=30, S=16$, system load $\alpha=1.875$, the rate $R_{1}=1 / 2,(23,35)_{8}$ convolutional 


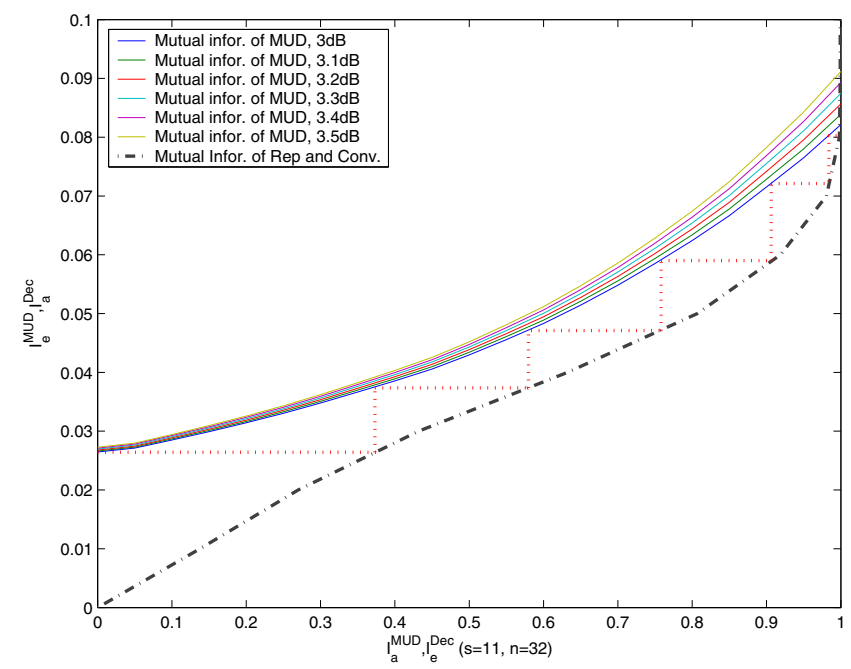

Fig. 2. EXIT chart with trajectory of PS-CDMA, $K=30, S=16$, information bits length $N_{b}=256$ bits, $E_{b} / N_{0}$ varies from $3 \mathrm{~dB}$ to $3.5 \mathrm{~dB}$ $(23,35)_{8}$ convolutional code with rate $R_{1}=1 / 2$, good scrambling sequences with $s=11, n=32$.

code at the transmitter, and the scrambling sequences based on algebraic-phase rotations. With $s=11, n=32$, and $J=2 \times 16 \times 256$, we can see this choice fulfills Lemmas 1 and 2. This implies that the matrix $\Phi$ is unitary.

In the following Section, using EXIT charts and trajectories, we analyze the iterative decoding convergency of the above example, and show that PS-CDMA using properly designed algebraic-phase scrambling sequences leads to better iterative decoding convergency, when compared to IDMA.

\section{EXIT CHARTS AND TRAJECTORIES, BER PERFORMANCE}

In the section, for PS-CDMA using different scrambling sequences and IDMA, we compare EXIT charts and trajectories, as well as BER performance.

Let $I_{a}$ and $I_{e}$ denote the a priori and extrinsic mutual information of the decoding processes. Assuming infinitely long codewords of each user, we have the following two EXIT curves:

1) $I_{e}^{M U D}=f^{M}\left(I_{a}^{M U D}, I_{c h}\right)$ describes the mutual information corresponding to the extrinsic output of the joint MUD and phase-remover process, given the a priori mutual information from MUD $I_{a}^{M U D}$ and from channel observation $I_{c h}$;

2) $I_{e}^{D e c}=f^{D}\left(I_{a}^{D e c}\right)$ describes the mutual information corresponding to the extrinsic output of the joint decoding process of the concatenated code.

Both computations of $f^{M}\left(I_{a}^{M U D}, I_{c h}\right)$ and $f^{D}\left(I_{a}^{D e c}\right)$ are given in $[12,13]$, respectively. Let $E_{b} / N_{0}$ be

$$
\frac{E_{b}}{N_{0}} \triangleq \frac{E_{s}}{N_{0}} \times \frac{1}{R_{1}}
$$

where $E_{b}$ and $E_{s}$ denote the system received energy-per-bit and energy-per-symbol, respectively.

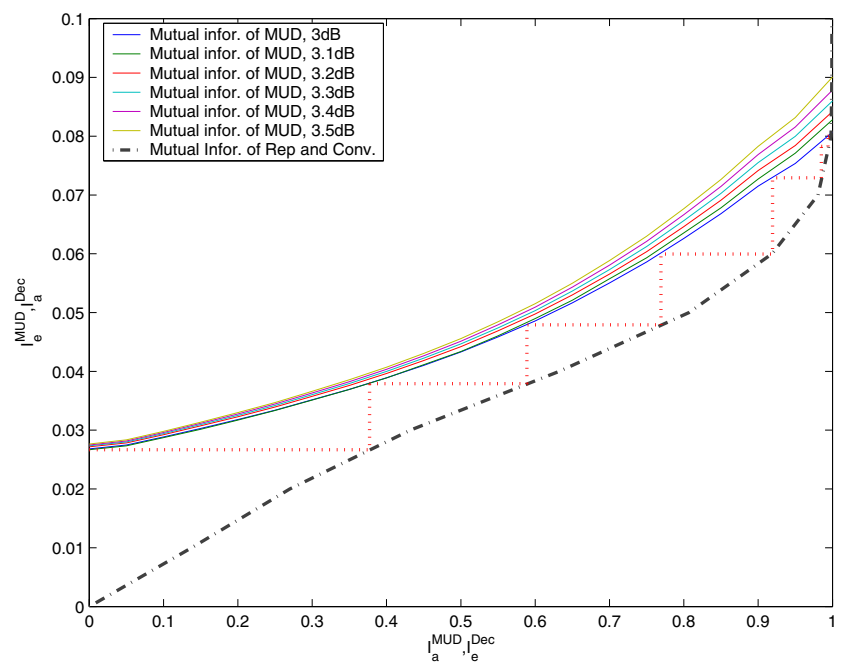

Fig. 3. EXIT chart with trajectory of PS-CDMA, $K=30, S=16$, scrambling sequences with random phases, $N_{b}=256$ bits, $E_{b} / N_{0}$ varies from $3 \mathrm{~dB}$ to $3.5 \mathrm{~dB},(23,35)_{8}$ convolutional code with rate $R_{1}=1 / 2$.

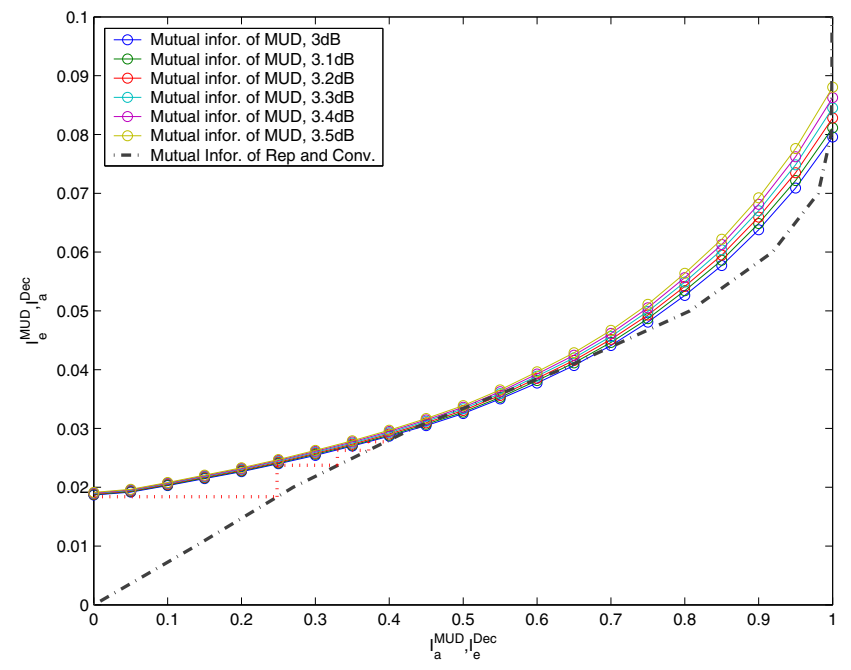

Fig. 4. EXIT chart with trajectory of IDMA, $K=30, S=16, N_{b}=256$ bits, $E_{b} / N_{0}$ varies from $3 \mathrm{~dB}$ to $3.5 \mathrm{~dB},(23,35)_{8}$ convolutional code with rate $R_{1}=1 / 2$.

Now we compare EXIT charts and trajectories of the overloaded PS-CDMA with properly designed scrambling sequences in the Example, PS-CDMA with random-phase scrambling sequences, and IDMA, respectively, when $E_{b} / N_{0}$ varies from $3 \mathrm{~dB}$ to $3.5 \mathrm{~dB}$. In EXIT chart computation, we use 50 blocks of information bits per user in both PS-CDMA and IDMA, respectively. We use the same system setting as that of the Example in Section III.

In Figs. 2 and 3 we compare the corresponding EXIT charts and the trajectories of PS-CDMA using algebraic scrambling sequences and random-phase scrambling sequences, respectively. In both figures, we present the EXIT curves for the 


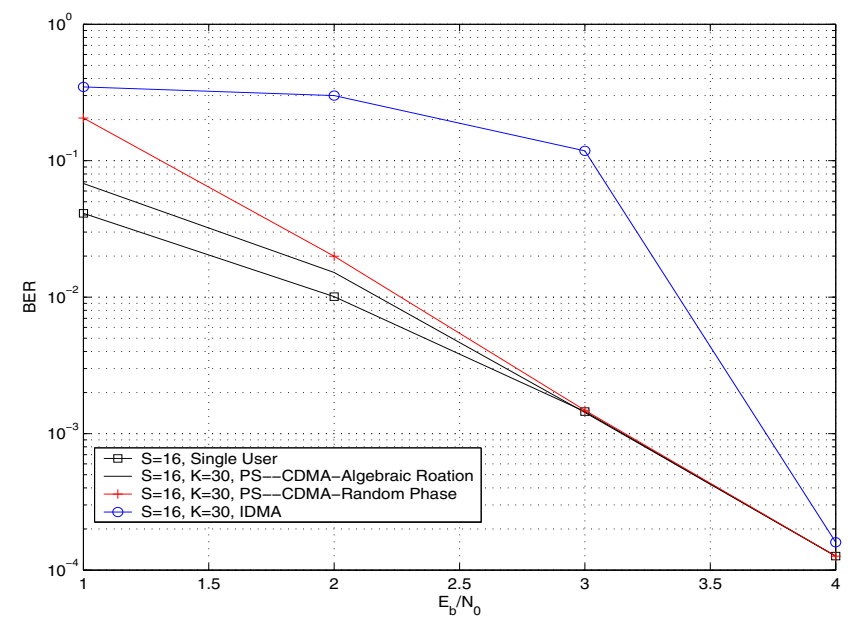

Fig. 5. Performance of PS-CDMA with the proposed scrambling sequences, random scrambling sequences, and IDMA, $N_{b}=256$ bits, $(23,35)_{8}$ convolutional code with rate $R_{1}=1 / 2, S=16, K=30$.

joint MUD and phase-remover process (solid line), the joint APP decoding of the repetition code and convolutional code (dashed-dotted line), respectively. We show their trajectories using dotted line. We observe that PS-CDMAs using both scrambling sequences have similar iterative decoding convergency, i.e., the joint iterative detection and decoding converges when $E_{b} / N_{0} \geq 3 \mathrm{~dB}$.

In Figs. 2 and 5 we compare iterative decoding convergency behaviors of PS-CDMA using properly designed algebraicphase scrambling sequences and that of IDMA. We see that the joint iterative detection and decoding of IDMA can not converge when $E_{b} / N_{0}$ varies from $3 \mathrm{~dB}$ to $3.5 \mathrm{~dB}$, while, in contrast, the PS-CDMA can converge.

In Fig. 5 we compare the BER performance of PSCDMA using properly designed algebraic-phase scrambling sequences, random-phase scrambling sequences and the IDMA. It is shown that PS-CDMA using properly designed algebraic-phase scrambling sequences has the best BER performance and converges to single user performance at the fastest speed.

\section{CONCLUSion}

In this paper, we consider complex scrambling sequences for overloaded PS-CDMA, where the information sequence of each user is encoded by a low-rate serially concatenated code (a convolutional code and a repetition code) and the iterative multiuser decoding MMSE-PIC scheme, proposed in $[5,8]$, was adopted at the receiver.

We design quasi-orthogonal complex algebraic-scrambling sequences specifically to mitigate MAIs for overloaded PSCDMA. We show that the PS-CDMA using properly designed algebraic-scrambling sequences has faster iterative decoding convergency and better BER performance, when compared to that of IDMA and PS-CDMA using random-phase scrambling sequences, respectively.
Finally, we remark that our orthogonal spreading sequence design can be easily adapted to any multiple access system with different number of users and various code rates.

\section{ACKNOWLEDGMENT}

The work of E. Viterbo was supported by the STREP project No. IST-026905 (MASCOT) within FP6 of the European Commission. The work of S. Shamai was supported by the European Commission in the framework of the FP7 Network of Excellence in Wireless COMmunications NEWCOM++. Dr Yi Hong's work was sponsored by The Royal Academy of Engineering, UK.

\section{REFERENCES}

[1] A.J. Viterbi, "Very low rate convolution codes for maximum theoretical performance of spread-spectrum multiple-access channels," IEEE J. Sel. Areas Commun., vol. 8, pp. 641-649, May 1990.

[2] J.Y.N. Hui, "Throughput analysis for code division multiple accessing of the spread spectrum channel," IEEE J. Sel. Areas Commun., vol. 8, pp. 482-486, July 1984

[3] P. K. Frenger, P. Orten, and T. Ottosson, "Code-spread CDMA with interference cancellation,” IEEE J. Sel. Areas Commun., vol. 17, pp. 20902095, Dec. 1999.

[4] P. K. Frenger, P. Orten, and T. Ottosson, "Code-spread CDMA using maximum free distance low-rate convolutional codes," IEEE Trans. Commun., vol. 48, pp. 135-144, Jan. 2000.

[5] X. Wang and H. V. Poor, "Iterative (turbo) soft interference calcellation and decoding for coded CDMA," IEEE Trans. Commun., vol. 47, pp. 1046-1061, Jul. 1999.

[6] S. Verdú and S. Shamai, "Spectral efficiency of CDMA with random spreading," IEEE Trans. Inf. Theory, vol. 45, pp. 622-640, Mar. 1999.

[7] R.H. Mahadevappa, J.G. Proakis, "Mitigating multiple access interference and intersymbol interference in uncoded CDMA systems with chiplevel interleaving," IEEE on Wireless Communications, vol. 1, no. 4, pp. 781-792, Oct. 2002

[8] L. Ping, L. Liu, K. Wu, and W. K. Leung, "Interleave Division MultipleAccess," IEEE Trans. Wireless Communications, vol. 5, no. 4, pp. 938947, April 2006.

[9] I. Oppenmann and B. Vucetic, "Complex spreading sequences with a wide range of correlation properties," IEEE Transactions on Communications, vol. 45, no. 3, Mar. 1997, pp. 365-375.

[10] I. Oppenmann, "Orthogonal complex-valued spreading sequences with a wide range ofcorrelation properties," IEEE Transactions on Communications, vol. 45, no. 11, Nov. 1997, pp. 1379-1380.

[11] R. Kohno, H. Fukumasa, H. Imai, "Polyphase Pseudo-Noise Sequences with Equivalent Even and Odd Correlation Properties," Proc. IEEE International Symposium on Information Theory, p. 404, Jan. 1993.

[12] S. ten Brink, "Convergence behavior of iteratively decoded parallel concatenated codes," IEEE Transactions on Information Theory, vol. 49, pp. 1727-1737, Oct. 2001.

[13] K. Li and X. Wang, "EXIT chart analysis of turbo multiuser detection," IEEE Transactions on Wireless Communications, vol. 4, no. 1, pp. 300311, Jan. 2005.

[14] F. Oggier, E. Viterbo, "Algebraic number Theory and code design for Rayleigh fading channels", Foundation and Trends in Communcations and Information Theory, vol. 1, pp. 333-415, 2004. 